\title{
Preparation, Characterization, and Antibacterial Activity of ZnS-NP's Filled Polyvinylpyrrolidone/Chitosan Thin Films
}

\author{
Menna A. Aboelwafa 1(D), Amr M. Abdelghany 2,* (D), Mahrous S. Meikhail ${ }^{1(\mathbb{D})}$ \\ Physics Department, Faculty of Science, Mansoura University, 355165, Mansoura, Egypt \\ Spectroscopy Department, Physics Division, National Research Centre, 33 El Behouth St., 12311, Dokki, Giza, Egypt \\ Correspondence: a.m_abdelghany@yahoo.com (A.M.);
}

Scopus Author ID 7003638023

Received: 2.02.2021; Revised: 2.03.2021; Accepted: 4.03.2021; Published: 10.03.2021

\begin{abstract}
Semi-natural polymeric Polyvinyl pyrrolidone/Chitosan (PVP/CS) blend containing gradient concentrations of $\mathrm{ZnS}$ nanoparticles (ZnS-NPs) were prepared using a simple casting route and characterized by using XRD, TEM, and FTIR analysis. The XRD diffraction peaks of the prepared samples show no distinct sharp bands that lead to the amorphous and homogeneous distribution of $\mathrm{ZnS}$ NPs inside the polymer composites. The TEM analysis showed the creation of $\mathrm{ZnS}$ nanoparticles of about uniform size and shape. Fourier transform infrared measurements (FTIR) take into account a detailed description of the different reaction mechanisms within the polymeric matrices.
\end{abstract}

Keywords: ZnS NP's; PVP/Chitosan; XRD; TEM; FTIR.

(C) 2021 by the authors. This article is an open-access article distributed under the terms and conditions of the Creative Commons Attribution (CC BY) license (https://creativecommons.org/licenses/by/4.0/).

\section{Introduction}

Semiconductor nanoparticles get a significant scientific effect because of their strong promise in optoelectronics and bio-applications [1]. Typically, the II-VI group's semiconductors are tested based on their special features rather than those of the other semiconductors. ZnS is a well-established broad bandgap semiconductor owing to its special optical and biocompatible properties. ZnS exists in 2 crystal structures, one in the cubic form (polymorphic stable at low temperature) and another in the hexagonal system (high temperature) [2]. ZnS shows a wide bandgap of nearly $3.91 \mathrm{eV}$ and $3.54 \mathrm{eV}$, respectively, for wurtzite and zinc blend (sphalerite) [3]. The characteristics of $\mathrm{ZnS}$ are very dependent on their size, structural form, and morphology. Wide bandgap II-VI semiconductors, particularly ZnS, are non-toxic, chemically more stable, and substantial phosphorus host materials used in optoelectronic applications such as solar cells transducers, photoconductors, light-emitting materials, optical coatings, sensors, and field-effect transistors [4, 5].

The helical polymer is chitosan that has gained a great deal of recognition as a biomaterial for implant applications and medical devices. Chitosan comes from chitin, a white, hard, inelastic, and nitrogenous polymer present in the exoskeleton of crustaceans, including insects, crawfish, shrimps, and crabs, in the internal structure of invertebrates, as well as in some fungi, where is the main fibrils polymer in the cell wall. It is the most available organic material, next only to the quantity of cellulose generated yearly by biosynthesis [6, 7]. Commercially, chitin is produced by the deacetylation process. While the degree of 
deacetylation of chitin achieves around 50 percent (relying on the polymer source), it is soluble in aqueous acidic media and is named chitosan. [8]. Chitin and chitosan are naturally available and renewable polymers that have remarkable biological activities, such as non-toxicity, hydrophilicity, biocompatibility biodegradability, cellular binding capability, adsorption, antimicrobial activity, and acceleration of wound healing, which accounts for their wide variety of application in food processing, cosmetics, agriculture, textile wastewater treatment, biotechnology, biomedical and pharmaceutical industries. Chitosan is well popular in biomedicine and is considered a potential candidate for many medical applications [9, 10].

Polyvinylpyrrolidone (PVP), often referred to as povidone or polyvidone, is created from its monomer N-vinylpyrrolidone [11]. Polyvinyl pyrrolidone (PVP) is an amorphous, synthetic polymer with elevated $\mathrm{Tg}$ values of up to $170{ }^{\circ} \mathrm{C}$ due to a solid pyrrolidone group, which is heavy with the drawing group and is known to form separate complexes with other polymers [12]. PVP is biodegradable, strong adhesion property, a stabilizing effect on suspensions and emulsions, and excellent solvent solubility in various polarities used in nanoparticle synthesis. PVP is a biocompatible polymer and non-toxic; it has also been classified as safe by the Food and Drug Administration (FDA) [13]. To this effect, PVP is widely used for biomedical and cosmetics uses, in addition to the food industry. Due to its amphiphilic, PVP can influence nanoparticles' structure and growth by retaining solubility in various solvents, controlled crystal growth, and discriminatory surface stabilization. PVP is one of the most promising polymers for nanogel preparation. Its flexible characteristics include water solubility, loss of toxicity, film formation, and adhesive strength [14].

\section{Materials and Methods}

\subsection{Materials used.}

Polyvinyl pyrrolidone (PVP) (purity >99\%) (MW 40.000) was extracted from (Bio Basic Canada INC.), Chitosan (Cs) with a deacetylation grade of $86 \%$ extra pure was extracted from the exporter's chemical laboratory Co. (Alpha Chemika, India). Zinc sulfide nanoparticles (ZnS-NPS) (MW $97.43 \mathrm{~g} / \mathrm{mol}$ ) were provided by the European Economic Community (EEC).

\subsection{Sample preparation.}

Samples analyzed were processed in thin membrane film using the conventional solvent casting and evaporation process. The estimated amount of Cs was dissolved by an intensive stirring $(50 \mathrm{~mL})$ of $2 \%$ aqueous acetic acid solution combined with a specific PVP amount and stirred for $60 \mathrm{~min}$. Then the combined solution was dropped on clean Petri dishes and dried at $50{ }^{\circ} \mathrm{C}$ for 24 hours. PVP/CS (80/20) blend was prepared with different concentrations of zinc sulfide nanoparticles (ZnS-NPS) loading, as seen in Table 1. Both samples were prepared along the same path, dropped on clean Petri dishes, and dried at $50^{\circ} \mathrm{C}$.

Table 1. Samples with varying concentrations of ZnS-NPs.

\begin{tabular}{c|c|c|c|c|c} 
Sample & S0 & S1 & S2 & S3 & S4 \\
\hline $\mathbf{Z n S}\left(\times \mathbf{1 0}^{-4} \mathbf{g}\right)$ & 0.00 & 2.50 & 5.00 & 10.0 & 20.0
\end{tabular}

\subsection{Physical measurement.}

X-ray diffraction scans (XRD) were obtained at Bragg's angle (20) ranging from (5-70) using (PAN analytical X'Pert PRO XRD system) operating at $30 \mathrm{kV}$ with $\mathrm{Cu} \mathrm{K} \alpha$ radiation 
(where, $\lambda=1.540 \AA$ ). The optical absorption spectra of FTIR were reported under the spectral spectrum of $2000-400 \mathrm{~cm}^{-1}$ using a single beam Nicolet is 10 spectrometers, absorption mode with 32 scans and a resolution of $2 \mathrm{~cm}^{-1}$ to analyze physical differences within the polymer matrix. A transmission electron microscope (JEOL-JEM-1011, Japan) was used to examine the size, diffusion, and structure of nanoparticles within the polymeric matrix.

\section{Results and Discussion}

\section{1. $X$-ray diffraction analysis (XRD).}

X-ray diffraction scan (XRD) is used to analyze the constructive spectroscopic method used to study homogeneity and overlapping of biopolymeric materials. It is a very helpful tool used to provide information on the composition (crystal/amorphous) of the lattice structure of the prepared substance and to provide very valuable information on the level of the crystalline sample of the synthesized thin film. XRD analysis occurs for pure samples and blend samples full of different concentrations of ZnS-NPs.

$\mathrm{X}$-ray spectrum for pure PVP, CS, and PVP/CS blended membranes represents in Figure 1. PVP provides 2 main peaks at $2 \theta$ equals $11^{\circ}$ and $21^{\circ}$, referred to as the amorphous nature of PVP $[15,16]$. Chitosan contained only one broad diffraction peak cantered at $2 \theta=$ $20.20^{\circ}$ due to (100) plane that shows the existence of an amorphous phase [17]. Pure (PVP/CS) blend film exhibits a broad peak of PVP at $2 \theta=21.7^{\circ}$, and a small hump was observed at around $2 \theta=10.5^{\circ}$ which suggests the amorphous nature of PVP.

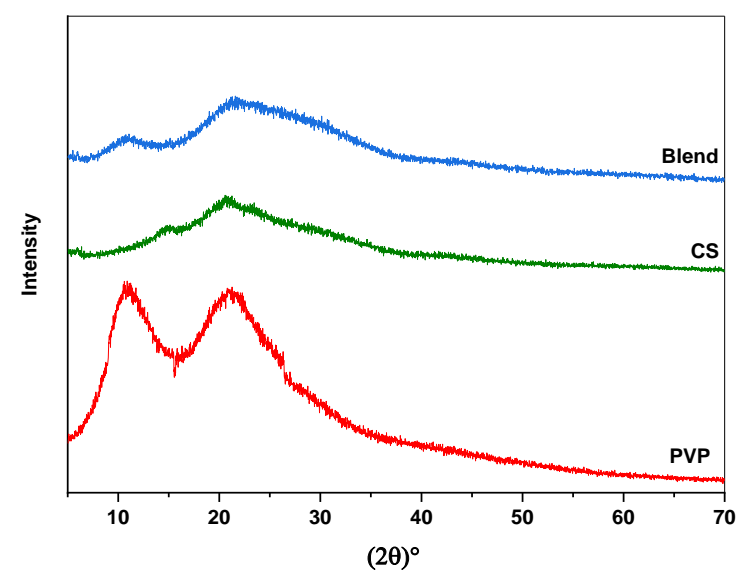

Figure 1. X-ray diffraction of pure PVP, pure CS, and PVP/CS blend.

XRD analysis of pure ZnS-NPs using the x-ray diffractometer shown in Figure 2. The wide-angle XRD results of the ZnS nanoparticles reveal the (100), (002), (112), (103), and (112) planes corresponding to $26.9,28.5,30.5,47.6$, and 56.4, respectively, indicating the hexagonal zinc wurtzite phase according to the [ JCPDS 05-0566]. The width of the peaks is due to the limited size of the crystals [18]. The nanocrystal size of $\mathrm{ZnS}$ was calculated through full-width at half maxima (FWHMs) using the Scherrer formula. The particle size of $\mathrm{ZnS}$ is estimated to be $39.4 \mathrm{~nm}$.

Figure 3 investigates XRD scans of the ZnS-NPs with different concentrations after mixing it in PVP/CS blend kept the amorphous form in the existence of PVP/CS blend until the concentration S3 of $\mathrm{ZnS}$ nanoparticles, three characteristic peaks of $\mathrm{ZnS}$ appear which mean that $\mathrm{ZnS}$ nanoparticles have an important influence on the amorphous component of the mixture and refer to completely interaction between blend and ZnS-NPs. 


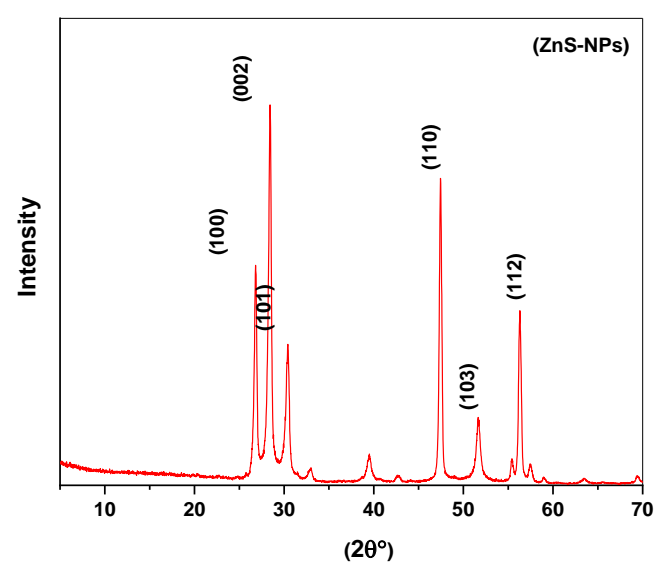

Figure 2. XRD pattern of pure ZnS-NPs.

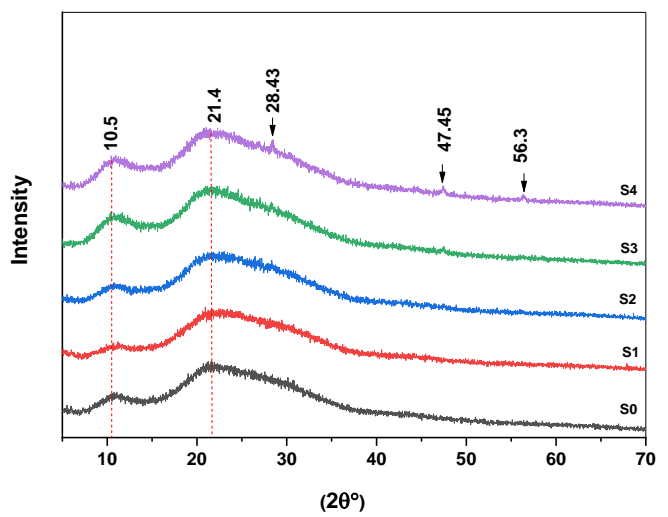

Figure 3. XRD of the blend (PVP/CS) and contain variable content of ZnS-nanoparticles.

\subsection{Transmission electron microscopy (TEM) study.}

TEM is a crucial instrument for direct imaging of nanomaterials to achieve quantitative measurements of morphology, and the estimated size of the as-prepared $\mathrm{ZnS}$ nanoparticles was analyzed in the TEM review. Figure 4(a) shows a TEM picture that clearly shows the distribution of a large number of $\mathrm{ZnS}$ nanoparticles distributed of a uniform shape ranging from polydisperse small spherical shapes to large spherical shapes with an average size of (40-60) $\mathrm{nm}$, which coincides with the findings of the XRD analysis [18]. The crystal structures of Wurtzite ZnS have been determined through HRTEM experiments. Figure 4(c) displays a representative HRTEM image of the wurtzite $\mathrm{ZnS}$ nanoparticle. The measured spacing of the crystallographic plane is $0.21 \mathrm{~A}$. The electron diffraction pattern of the nanoparticles compatible with the ZnS hexagonal crystal (JCPDS 05-0566) is shown in Figure 4(b).
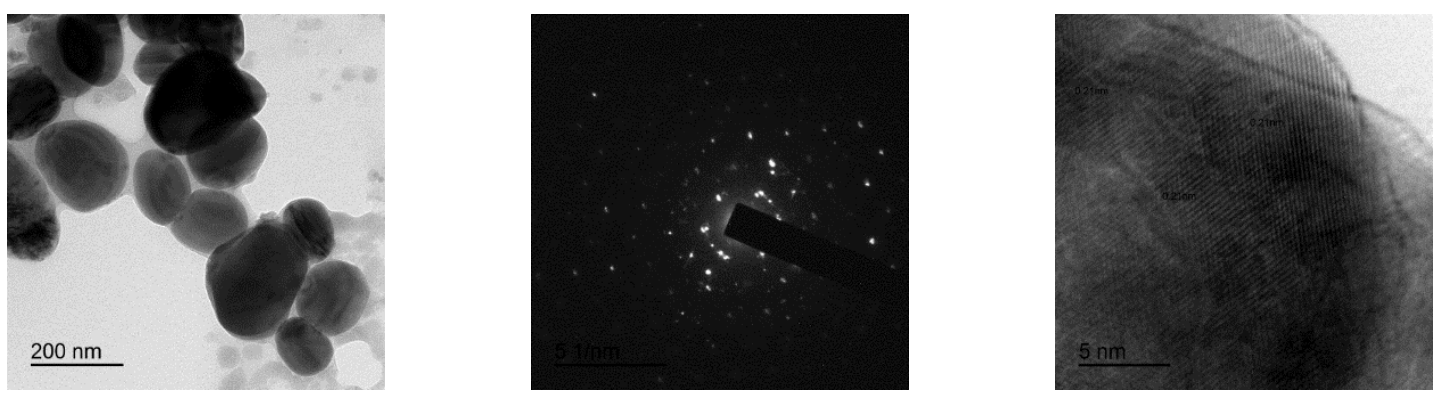

Figure 4. (a) TEM image of ZnS NP's; (b) diffraction pattern; (c) HRTEM showing lattice spacing.

\subsection{Fourier transforms infrared analysis (FTIR).}


FT-IR spectroscopy is a very effective technique for recognizing vibration bands related to major chemical groups, identifying molecular structure, which includes the identification of bands for each sample and the inter-molecular contact between polymers. Figure 5 displays FT-IR absorbance spectra in the range $2000-400 \mathrm{~cm}^{-1}$ of pure PVP, CS, and CS/PVP blend films at room temperature.

For pure PVP, the spectra display peaks at $567 \mathrm{~cm}^{-1}$ and $655 \mathrm{~cm}^{-1}$ lead to N-C=O $[15$, 16]. The $750 \mathrm{~cm}^{-1}$ band correlates to the $\mathrm{C}-\mathrm{C}$ chain. Besides, bending vibrations at $1291 \mathrm{~cm}^{-1}$, which are related to the pyrrolidone structure of the $\mathrm{C}-\mathrm{N}$, can be established. It is recalled that PVP is only a bi-substituted amide, the characteristic absorption of amines at approximately $3400-3500 \mathrm{~cm}^{-1}$ has not been established $[15,16]$. The stretching vibration around $1662 \mathrm{~cm}^{-1}$ could be related to $\mathrm{C}=\mathrm{O}$ in the pyrrolidone group. The peaks at $1370 \mathrm{~cm}^{-1}$ and $1446 \mathrm{~cm}^{-1}$ often apply to the $\mathrm{CH}$ deformation modes of the $\mathrm{CH} 2$ group [16].

For pure CS bands at $665 \mathrm{~cm}^{-1}, 1035 \mathrm{~cm}^{-1}$, and $1076 \mathrm{~cm}^{-1}$ are indicative of its saccharide structure (skeletal vibrations involving C-O stretching), absorption bands at $1160 \mathrm{~cm}^{-1}$ related to anti-symmetric stretching (C-O-C bridge), coupling results of angular deformation $(\mathrm{N}-\mathrm{H})$ and axial stretching at $1426 \mathrm{~cm}^{-1}$ and $1382 \mathrm{~cm}^{-1}$, the peak at $1574 \mathrm{~cm}^{-1}$ is related to $(\mathrm{NH})$ bending (amide I) (NH2) and the peak $1653 \mathrm{~cm}^{-1}$ corresponding to $\mathrm{C}=\mathrm{O}$ stretching (amide II) $\mathrm{O}=\mathrm{C}-\mathrm{NHR}[17,19]$.

For the PVP/CS blend, the peaks at $1035 \mathrm{~cm}^{-1}$ and $1076 \mathrm{~cm}^{-1}$ (skeletal vibrations including $\mathrm{C}-\mathrm{O}$ stretching) are typical of the saccharide frame. The peak at $1291 \mathrm{~cm}^{-1}$ was leading to $\mathrm{C}-\mathrm{N}$ stretching vibration, which suggests the amine group's existence in the pure membrane matrix. The peak at $1430 \mathrm{~cm}^{-1}$ is the product of the pairing of N-H angular deformation and $\mathrm{C}-\mathrm{N}$ axial deformation.

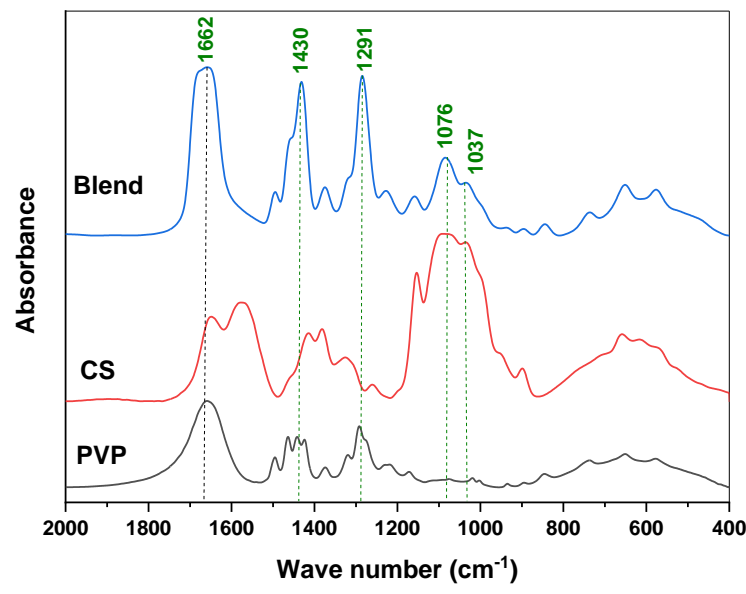

Figure 5. FT-IR absorption spectra of the pure blend, pure PVP, and pure CS.

Figure 6 reveals FTIR absorption in the area of $2000-400 \mathrm{~cm}^{-1}$ by comparing the spectra of a blend (PVP/CS) when blending with different concentrations of zinc sulfide nanoparticles (ZnS-NPs) shows that the spectral changes appear as a result of $\mathrm{ZnS}$ blending. There was a strong shift in severity with a rise in the content of $\mathrm{ZnS}$ doping. The peaks at $1434 \mathrm{~cm}^{-1}$ and $1283 \mathrm{~cm}^{-1}$ are broad and decrease in intensity with increasing ZnS-NPS concentration up to $(0.025 \%)$. The band at $1662 \mathrm{~cm}^{-1}$ decreases in intensity, and the band's width increases with an increase in the concentration of ZnS-NPs that indicates the complexation between ZnS-NPs and (PVP/CS) polymer blend by a coordinate bond. 


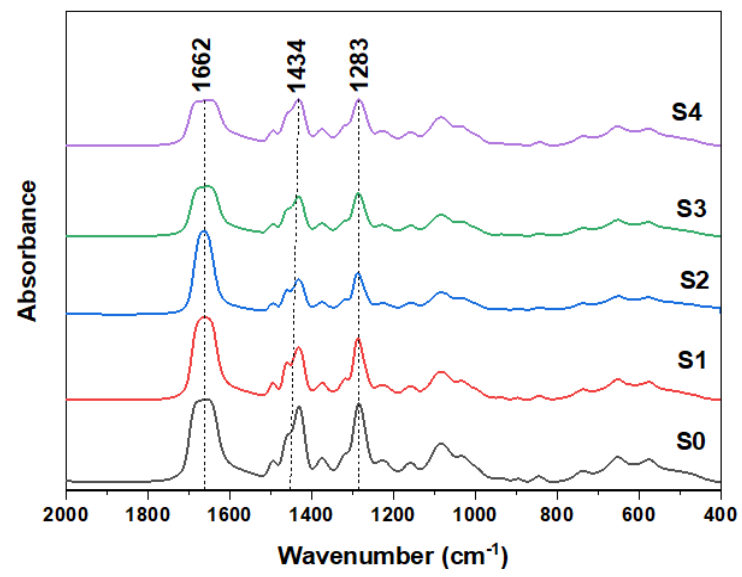

Figure (6) FT-IR absorption spectra of pure blend and biosynthesized ZnS-NPs.

\subsection{Antimicrobial activity.}

Zinc sulfide exhibits a wide-spectrum biocide behavior towards multiple viruses, bacteria, and fungi $[20,21]$. The antimicrobial behavior of sampled ZnS-NPs with varying concentrations in the polymer blend was then checked for the compounds' antimicrobial activity towards gram-negative E. Coli (Escherichia coli) and gram-positive S. aureues (Staphylococcus aureus). Specimens were planted in agar-containing dishes (beef extract $3 \mathrm{~g}$ + agar $20 \mathrm{~g}+$ peptone $5 \mathrm{~g}$ ), and Petri dishes were cultivated at $36{ }^{\circ} \mathrm{C}$. After 24 hours of incubation, inhibition was recorded.

This is well reported that if an antimicrobial material interaction with the bacterial strain, a specific area from around the antibacterial material is formed (inhibition zone). The strain susceptible to the antibacterial agent has a wider diameter, while the resistance strain has a smaller inhibition area diameter. PVP/CS blend was observed to exhibit no inhibition zone, signaling a lack of antibacterial activity in both bacterial species, primarily due to inadequate $\mathrm{ZnS}$ nanoparticles. From either side, PVP/CS/ZnS clearly showed a distinct area of the inhibition zone in both bacterial strains.

Figure 7 reveals an increase in an inhibition zone associated with the increase in the concentration of ZnS-NP's content.

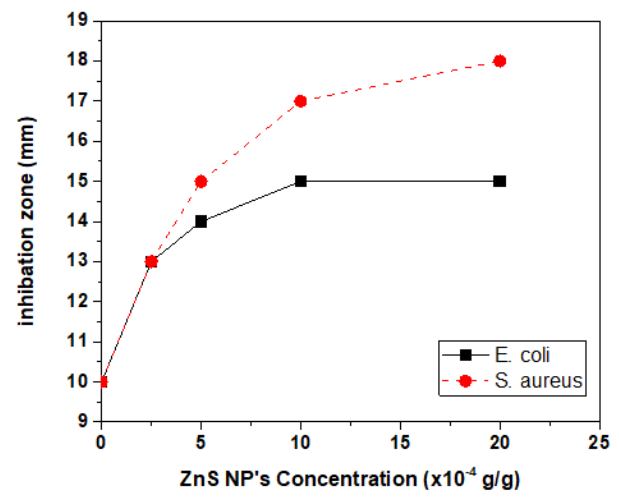

Figure 7. Variation of the inhibition zone associated versus concentration of ZnS-NP's.

The basic method for the antibacterial activity of PVP/CS/ZnS may be clarified. ZnS, a semiconductor material, can produce biologically reactive oxygen species (ROS) that induce hydroxyl radicals $\left(\mathrm{OH}^{*}\right)$ under UV-light irradiation because of its high bandgap. Even after that, this study aims to produce ROS by $\mathrm{ZnS}$ indoor light activation. Holes $(\mathrm{h}+)$ and electrons (e-) are generated in the valence band (VB) and the conduction band (CB) during the activation 
process. By indoor light activation, the VB holes are captured by water $\left(\mathrm{H}_{2} \mathrm{O}\right)$ or hydroxyl groups $(\mathrm{OH})$ on the layer of the $\mathrm{ZnS}$ to produce hydroxy radicals $\left(\mathrm{OH}^{\circ}\right)$. Besides this, the extreme conductivity in polymers (caused by the presence of active $\mathrm{CH}_{3}$ groups) plays a crucial part in transporting exciting electrons from the $\mathrm{ZnS} \mathrm{CB}$ to the film surface in which they interact with dissolved oxygen molecules to produce superoxide radical ions $\left(\mathrm{O}_{2}{ }^{\circ}\right)$. After protonation, $\mathrm{O}_{2}{ }^{\circ}$ produces hydroperoxide radicals $\left(\mathrm{HO}_{2}{ }^{\circ}\right)$ that contribute to the creation of $\mathrm{OH}^{*}$. $\mathrm{OH}^{*}$ generated are therefore capable of helping to kill the bacterial cell membrane that contributes to cell function loss and cell death [22].

\section{Conclusions}

Semi-natural polymer blend \%80PVP/20\%CS and other samples of the same composition containing minor doping fractions of zinc sulfide nanoparticles were successfully synthesized via a traditional casting routine. XRD pattern shows the crystalline nature of used zinc sulfide and the amorphous nature of other pristine polymers. Obtained diffraction data suggest that ZnS-NP's takes an interstitial position within the polymeric matrix. FTIR shows prominent peaks attributed to the vibrational groups within both PVP and Cs polymer and points out the complexation between polymers and zinc sulfide. TEM images show a uniform distribution of nanoparticles within the polymeric matrix. Synthesized samples show an increase in antibacterial effects against gram-positive and gram-negative pathogenic bacteria that increase with increasing nanoparticle content.

\section{Funding}

This research received no external funding.

\section{Acknowledgments}

This research has no acknowledgment.

\section{Conflicts of Interest}

The authors declare no conflict of interest.

\section{References}

1. Ajibade, P.A.; Oluwalana, A.E.; Sikakane, B.M.; Singh, M. Structural, photocatalytic and anticancer studies of hexadecylamine capped ZnS nanoparticles. Chem. Phys. Lett. 2020, 755, 137813, https://doi.org/10.1016/j.cplett.2020.137813.

2. Heiba, Z.K.; Mohamed, M.B. Structural, Optical and Magnetic Properties of ZnS Co-doped with Cd and Fe. Journal of Inorganic and Organometallic Polymers and Materials 2020, 30, 879-888, https://doi.org/10.1007/s10904-019-01226-9.

3. Nakamura, Y.; Iso, Y.; Isobe, T. Bandgap-Tuned CuInS2/ZnS Core/Shell Quantum Dots for a Luminescent Downshifting Layer in a Crystalline Silicon Solar Module. ACS Applied Nano Materials 2020, 3, 3417-3426, https://doi.org/10.1021/acsanm.0c00175.

4. Shalaan, E.; Ibrahim, E.; Al-Marzouki, F.; Al-Dossari, M. Observation of mixed types of energy gaps in some II-VI semiconductors nanostructured films: towards enhanced solar cell performance. Appl. Phys. A 2020, 126, 852, https://doi.org/10.1007/s00339-020-04045-9.

5. Woods-Robinson, R.; Han, Y.; Zhang, H.; Ablekim, T.; Khan, I.; Persson, K.A.; Zakutayev, A. Wide Band Gap Chalcogenide Semiconductors. Chem. Rev. 2020, 120, 4007-4055, https://doi.org/10.1021/acs.chemrev.9b00600. 
6. Lim, C.; Hwang, D.S.; Lee, D.W. Intermolecular interactions of chitosan: Degree of acetylation and molecular weight. Carbohydr. Polym. 2021, 259, 117782, https://doi.org/10.1016/j.carbpol.2021.117782.

7. Kanaan, A.F.; Piedade, A.P.; de Sousa, H.C.; Dias, A.M.A. Semi-interpenetrating chitosan/ionic liquid polymer networks as electro-responsive biomaterials for potential wound dressings and iontophoretic applications. Materials Science and Engineering: C 2021, 121, 111798, https://doi.org/10.1016/j.msec.2020.111798.

8. López-Valverde, N.; López-Valverde, A.; Ramírez, J.M. Systematic Review of Effectiveness of Chitosan as a Biofunctionalizer of Titanium Implants. Biology 2021, 10, https://doi.org/10.3390/biology10020102.

9. Pita-López, M.L.; Fletes-Vargas, G.; Espinosa-Andrews, H.; Rodríguez-Rodríguez, R. Physically crosslinked chitosan-based hydrogels for tissue engineering applications: A state-of-the-art review. Eur. Polym. J. 2021, 145, 110176, https://doi.org/10.1016/j.eurpolymj.2020.110176.

10. Neacsu, I. A., Serban, A. P., Nicoara, A. I., Trusca, R., Ene, V. L., \& Iordache, F. Biomimetic Composite Scaffold Based on Naturally Derived Biomaterials. Polymers, 2020, 12(5), 1161 . doi: 10.3390/polym12051161, eISSN: 2073-4360, WOS:000541431100166

11. Dispenza, C.; Sabatino, M.A.; Grimaldi, N.; Dahlgren, B.; Al-Sheikhly, M.; Wishart, J.F.; Tsinas, Z.; Poster, D.L.; Jonsson, M. On the nature of macroradicals formed upon radiolysis of aqueous poly $(\mathrm{N}-$ vinylpyrrolidone) solutions. Radiat. Phys. Chem. 2020, 174, 108900 , https://doi.org/10.1016/j.radphyschem.2020.108900.

12. Zhao, M.; Tang, Z.; Zhang, X.; Li, Z.; Xiao, H.; Zhang, M.; Liu, K.; Ni, Y.; Huang, L.; Chen, L.; Wu, H. A self-healing, stretchable, and conductive Poly(N-vinylpyrrolidone)/gallic acid composite hydrogel formed via hydrogen bonding for wearable electronic sensors. Composites Sci. Technol. 2020, 198, 108294, https://doi.org/10.1016/j.compscitech.2020.108294.

13. Mohamed Azharudeen, A.; Karthiga, R.; Rajarajan, M.; Suganthi, A. Selective enhancement of nonenzymatic glucose sensor by used PVP modified on $\alpha$-MoO3 nanomaterials. Microchem. J. 2020, 157, 105006, https://doi.org/10.1016/j.microc.2020.105006.

14. Kuźmińska, A.; Butruk-Raszeja, B.A.; Stefanowska, A.; Ciach, T. Polyvinylpyrrolidone (PVP) hydrogel coating for cylindrical polyurethane scaffolds. Colloids Surf. B. Biointerfaces 2020, 192, 111066, https://doi.org/10.1016/j.colsurfb.2020.111066.

15. Farea, M.O.; Abdelghany, A.M.; Oraby, A.H. Optical and dielectric characteristics of polyethylene oxide/sodium alginate-modified gold nanocomposites. RSC Advances 2020, 10, 37621-37630, https://doi.org/10.1039/D0RA07601E.

16. Abdelghany, A.M.; Farea, M.O.; Oraby, A.H. Structural, optical, and electrical reinforcement of gammairradiated PEO/SA/Au NPs nanocomposite. Journal of Materials Science: Materials in Electronics 2021, https://doi.org/10.1007/s10854-021-05371-1.

17. Abdelghany, A.M.; Ayaad, D.M.; Aboelkheir, A.M. The effects of prolonged UV irradiation on the physicochemical characteristics of chitosan lamellar films modified with nanoparticulate silver vanadate nanorods. Polym. Bull. 2020, 77, 5489-5503, https://doi.org/10.1007/s00289-019-03029-x.

18. Mansour, A.F.; Abdo, M.A.; Maged, F.A.; Agag, G.M. Synthesis, Optical Properties and Stabilization of ZnS Quantum Dots by Polymeric Matrices. Journal of Inorganic and Organometallic Polymers and Materials 2021, 10.1007/s10904-021-01884-8, https://doi.org/10.1007/s10904-021-01884-8.

19. Cirri, M.; Maestrelli, F.; Scuota, S.; Bazzucchi, V.; Mura, P. Development and microbiological evaluation of chitosan and chitosan-alginate microspheres for vaginal administration of metronidazole. Int. J. Pharm. 2021, 598, 120375, https://doi.org/10.1016/j.ijpharm.2021.120375.

20. Jin, S.-E.; Jin, H.-E. Antimicrobial Activity of Zinc Oxide Nano/Microparticles and Their Combinations against Pathogenic Microorganisms for Biomedical Applications: From Physicochemical Characteristics to Pharmacological Aspects. Nanomaterials 2021, 11, https://doi.org/10.3390/nano11020263.

21. Liu, Y.; Huang, J.; Feng, X.; Li, H. Thermal-Sprayed Photocatalytic Coatings for Biocidal Applications: A Review. J. Therm. Spray Technol. 2020, 10.1007/s11666-020-01118-2, https://doi.org/10.1007/s11666-02001118-2.

22. Panthi, G.; Ranjit, R.; Khadka, S.; Gyawali, K.R.; Kim, H.-Y.; Park, M. Characterization and antibacterial activity of rice grain-shaped $\mathrm{ZnS}$ nanoparticles immobilized inside the polymer electrospun nanofibers. Advanced Composites and Hybrid Materials 2020, 3, 8-15, https://doi.org/10.1007/s42114-020-00141-9. 